Ann. Biol. anim. Bioch. Biophys., Ig62, 2 (2), I43-I49

\title{
L'UTILISATION DIGESTIVE RÉELLE DU PHOSPHORE DU FOIN DE LUZERNE PAR LE MOUTON, MESUREE A L'AIDE DE ${ }^{32} P$
}

\author{
L. GUEGUEN \\ avec la collaboration technique de Michelle Frasvier-LANGlois \\ Station de Biochimie et de Nutrition, \\ Centre national de Recherches zootechniques, Jouy-en-Josas (Seine-et-Oise)
}

\section{SOMMAIRE}

L'excrétion fécale endogène de phosphore a été estimée chez trois béliers adultes à l'aide d'une méthode de dilution isotopique simplifiée. Des bilans de phosphore ont été effectués à partir du $6^{\mathrm{e}}$ jour après une injection unique de ${ }^{32} \mathrm{P}$, et les radioactivités spécifiques du phosphore du plasma et des fèces ont été comparées.

Les évolutions des activités spécifiques du phosphore du plasma et des fèces sont remarquablement parallèles entre animaux.

En moyenne 60 p. Ioo du phosphore fécal est endogène. Ainsi, le coefficient d'utilisation digestive réelle du phosphore du foin de luzerne distribué est de $55 \mathrm{p}$. 100, tandis que son utilisation digestive apparente est négative.

L'excrétion fécale endogène de phosphore est de $2, \mathrm{r} \mathbf{g}$ par jour et peut être assimilée au besoin d'entretien puisque les pertes urinaires sont négligeables. Pour couvrir les besoins d'entretien il faudrait donc apporter $3,8 \mathrm{~g}$ de phosphore par jour. Ceci correspond à $42 \mathrm{mg}$ de phosphore par $\mathrm{kg}$ de poids vif de l'animal.

Les expériences classiques de bilans ne permettent d'évaluer que l'utilisation digestive apparente et la rétention apparente du phosphore de la ration. Pour estimer avec certitude l'utilisation digestive réelle du phosphore il est nécessaire de connaître la fraction endogène, déjà utilisée à des fins métaboliques, du phosphore excrété par les fèces. En effet, le phosphore réexcrété dans l'intestin et provenant de l'organisme se retrouve dans les fèces au même titre que le phosphore non digéré de la ration.

Le coefficient d'utilisation digestive réelle est donc ainsi défini :

$$
\text { C. U. D. réel }=\frac{\mathrm{P}_{i}-\left(\mathrm{P}_{f}-\mathrm{P}_{f e}\right)}{\mathrm{P}_{i}} \times 100,
$$

sachant que $\mathrm{P}_{i}$ est la quantité de phosphore ingérée, $\mathrm{P}_{f}$ la quantité de phosphore excrétée par les fèces et $P_{s u}$ la partie endogène du phosphore fécal. 
L'estimation de l'utilisation digestive et métabolique réelle du phosphore est particulièrement importante pour calculer, à partir des besoins nets (entretien, croissance, lait), les apports de phosphore recommandés pour un état physiologique déterminé. De même, pour évaluer l'efficacité biologique de plusieurs sources de phosphore il est nécessaire de comparer les digestibilités réelles. En outre, chez les ruminants adultes oì l'excrétion urinaire de phosphore est négligeable, l'excrétion endogène fécale de phosphore constitue la totalité des pertes métaboliques et peut donc être assimilée au besoin d'entretien (dans la mesure où ce besoin est constant).

L'excrétion fécale endogène de phosphore peut être facilement estimée, dans des conditions physiologiques normales, par 1'emploi de la méthode de dilution isotopique. Cette technique, employée pour la première fois par Hevesx (1939) a été mise au point et perfectionnée par KI,EIBER et al. (I95I) puis par LOFGREEN et KLEIBER (I954) pour l'étude de l'excrétion fécale endogène de phosphore.

PRIXCIPE DE LA MÉTHODE

En injectant à l'animal une solution contenant ${ }^{32} \mathrm{P}$ on obtient, au bout de quelques jours, un état stationnaire tel que le taux de sécrétion de ${ }^{32} \mathrm{P}$ dans l'intestin demeure constant. KLEIBER et $a l$. (I95I) obtenaient cet état d'équilibre en effectuant des injections bi-quotidiennes durant $\mathrm{I} 2$ jours.

On admet alors que, dans cet état d'éruilibre, le phosphore du plasma et le phosphore endogène sécrété dans l'intestin ont la même radioactivité spécifique. Sachant que toute la radioactivité apparaissant dans les fèces résulte de l'excrétion endogène de $P$, il est facile de calculer la fraction endogène $f_{e}$, connaissant les activités spécifiques de $\mathrm{P}$ du plasma, $A_{p}$, et des fèces, $A_{\mathrm{F}}$, ainsi que la quantité totale de $P$ excrétée par les fèces, $F$.

$\mathrm{A}_{p} \cdot f_{e}=\mathrm{A}_{\mathrm{F}} \cdot \mathrm{F}$.

$\mathrm{I}_{\text {a }}$ proportion de $\mathrm{P}$ endogène dans le $\mathrm{P}$ fécal total est donc donnée par le rapport des radioactivités spécifiques de $P$ des fèces et du plasma.

'Toutefois, il est nécessaire que les quantités journalières ingérées demeurent constantes durant l'expérience, de telle façon que la dilution du phosphore endogène marqué par le phosphore stable d'origine alimentaire soit constante.

LOFGREEN et KLEIBER (I954) ont considérablement simplifié cette méthode en montrant qu'il suffisait de faire une seule injection sous-cutanée au début de l'expérience et que l'on obtenait, après le $4^{\mathrm{e}}$ jour, une parfaite corrélation entre les radioactivités spécifiques de $\mathrm{P}$ du plasma et des fèces (radioactivités spécifiques qu'il n'est donc pas nécessaire de maintenir constantes). De plus, la relation entre les radioactivités spécifiques de $\mathrm{P}$ du plasma et des fèces d'une part et le temps d'autre part, est exponentielle du $4^{\mathrm{e}}$ au $7^{\mathrm{e}}$ jour et linéaire du $7^{\mathrm{e}}$ au $\mathrm{I} 4^{\mathrm{e}}$ jour. Il suffirait donc, en utilisant cette période de décroissance linéaire, de prélever du sang le $7^{\mathrm{e}}$ et le $13^{\mathrm{e}}$ jour et de confectionner un échantillon moyen des fèces recueillis du $9^{\mathrm{e}}$ au $15^{\mathrm{e}}$ jour, compte tenu d'un décalage de 2 jours entre plasma et fèces (LOFGREEN I960).

Nous avons adopté cette dernière technique, mais en effectuant, à titre de vérification, plusieurs prélèvements de sang et d'excreta durant la période utilisée. 


\title{
MATÉRIEI EXPÉRIMENTAL ET MÉTHODES UTILISÉES
}

\author{
I" Animanx et alimentation
}

L'expérience a porté sur 3 béliers, de race lle de lirance, âgés de 3 ans et demi à 4 ans et pesant de 85 à $90 \mathrm{~kg}$.

Les animaux ont été nuaintenus en cages à métabolismes (permettant de séparer les urines et les féces) durant 6 jours avant le début de l'expérience, afin d'obtenir un niveau d'ingestion pratiquement constant.

TABLEAU I

Composition minérale moynne du foin do luserne distribue et des refus, en g par $\mathrm{kg}$ de $\mathrm{M}$. S.

\begin{tabular}{|c|c|c|c|c|c|}
\hline Élément & $\mathrm{P}$ & $\mathrm{Ca}$ & $\mathrm{K}$ & $\mathrm{Na}$ & $\mathrm{Mg}$ \\
\hline Foin de luzeme. & $\because, 19$ & $11, \overrightarrow{7}$ & $L^{\prime} \mathbf{i}, \bar{i}$ & 1,is & 2,18 \\
\hline Refus ........ & 1,62 & 9,2 & 12,6 & 1,12 & 1,60 \\
\hline
\end{tabular}

Le foin de luzene, dont la composition minérale movenne est indiquée dans le tableau I, a été distribué deux fois par jour, avec de l'eau à volonté. Les quantités distribuées et les refus ont été pesés charque jour pour charque animal.

\section{Déroulement de lexpérience}

Les 3 béliers $\mathrm{A}, \mathrm{B}, \mathrm{C}$, unt reçu respectivement par injection sous-cutanée (à l'intérieur de la cuisse), environ $4,5-3,0$ et $4.5 \mathrm{mlC}$ de ${ }^{32} \mathrm{P}$ sous forme de solution neutre stérile isotonique de $\mathrm{PO}_{4} \mathrm{INNa}_{2}$.

Pour éviter des contaminations importantes par les fortes excrétions de radioactivité se produisant durant les premiers jours, les planchers des cages à métabolismes et les récipients de récolte des excreta ont été renouvelés arant la première période de bilans.

Des prises de sang ont été effectuées dans la veine jugulaire gauche le $6^{\mathrm{e}}$, le $9^{\mathbf{e}}$, le $\mathbf{z}^{\mathbf{e}}$ et le I $9^{\mathrm{e}}$ jour après l'injection, tandis que des bilans complets étaient réalisés durant des périodes de 24 heures du $8^{\text {e }}$ au $2 \mathrm{I}^{\mathrm{e}}$ jour.

\section{$3^{\circ}$ Préparation des échantillons et míthodes de dosage}

Le sang, recueilli sur héparine, a été centrifugé imınédiatement pour séparer le plasma.

Pour le sang et l'urine, les comptages ont été effectués sur des prises de 1 ou $2 \mathrm{ml}$ placées dans des coupelles en aluminium et séchées sous une lampe à infra rouge. Le dosage de $\mathrm{P}$ a été effectué après minéralisation nitro-perchlorique d'une prise de $10 \mathrm{ml}$.

Pour les fèces, une nise en solution a été effectuée avant le comptage. lin effet, des mesures relatives suffisent pour déterminer le rapport des activités spécifiques de $\mathbf{P}$ du plasma et des fèces, à condition que les géométries de comptage soient rigoureusement identiques. Ainsi, une prise de $20 \mathrm{~g}$ de fèces frais a été effectuée à partir d'un échantillon représentatif de $200 \mathrm{~g}$ et a étébroyée dans un mixer Waring-Blendor (les échantillons étant broyés dans des pots différents) avec $180 \mathrm{ml}$ d'eau distillée. Puis, environ $40 \mathrm{~g}$ du broyat homogénéisé ont été séchés sur un bain de sable dans une capsule en silice et incinérés au four à $530.550^{\circ} \mathrm{C}$. Les cendres ont été reprises par $\mathrm{HCl}$ pur, puis par $\mathrm{HNO}_{3}$ à Io p. Ioo sur un bain de sable et diluées convenablement. Les dosages de $\mathrm{P}$ et les comptages ont été effectués sur des prises aliquotes de cette solution.

Le dosage de $\mathrm{P}$ dans les fourrages a été effectué après incinération au four de $\mathrm{g}$ de produit sec et mise en solution comme dans le cas des fèces.

Les dosages de $\mathrm{P}$ ont été effectués par colorimétrie du phosphovanadomolybdate d'ammonium et les comptages de radioactivité à l'aide d'un compteur Geiger-Müller éruipé d'un passeur automatique d'échantillons. T.e mouvement propre très réduit ( ${ }_{3}$ chocs $/ \mathrm{mn}$ ) du compteur a permis de mesurer des activités faibles (taux de comptage de 50 à 500 chocs $/ \mathrm{mn}$ avec prédétermination de $\mathrm{I}$ ooo chocs pour toutes les mesures).

Les activités des échantillons de fèces ont été corrigées pour tenir compte du décalage de 2 jours avec les prélèvements de sang correspondants. 


\section{RÉSULTATS}

$\mathrm{I}^{\circ}$ Évolution des radioactivités spécifiques du phosphore du plasma et des fèces

Pour les trois animaux, les évolutions des radioactivités spécifiques de $\mathrm{P}$ du plasma et des fèces sont remarquablement similaires, compte tenu du fait que le bélier B n'a reçu que $3,0 \mathrm{mC}$ au lieu de $4,5 \mathrm{mC}$ pour les deux autres (fig. I).

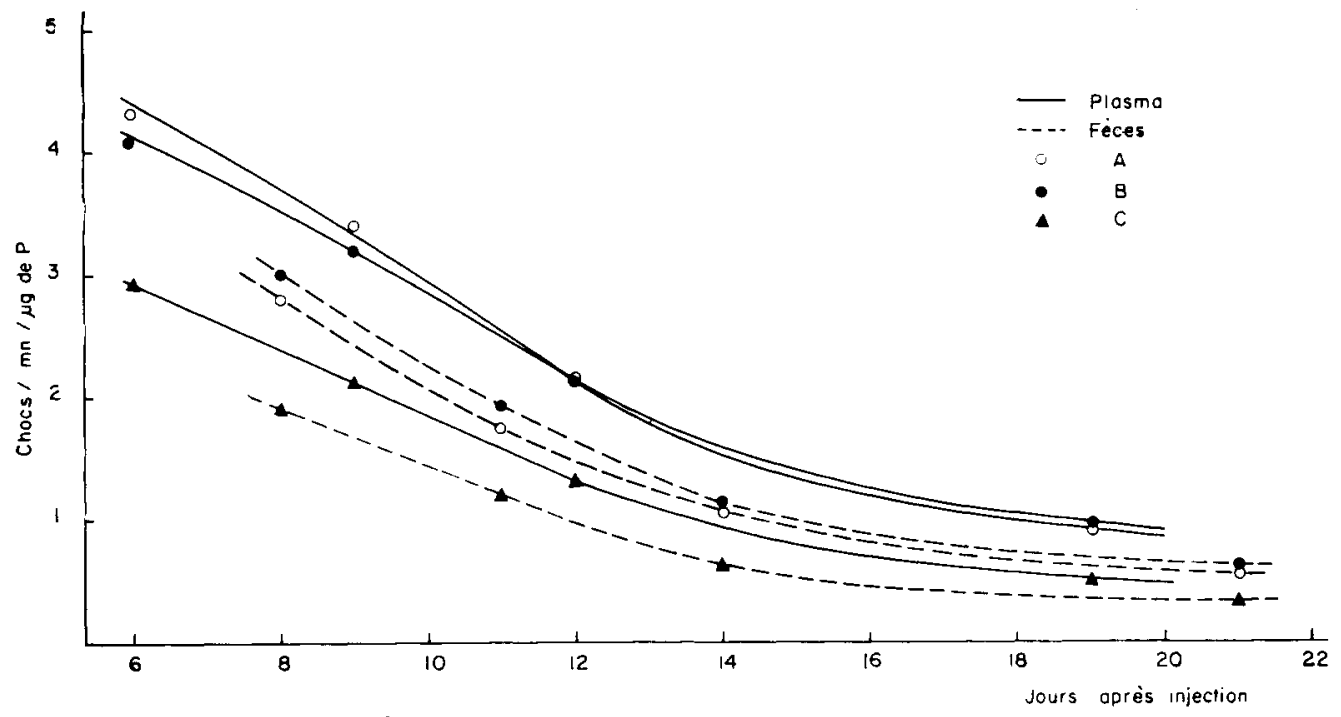

FIG. I. - Evolution des radioactivités spécifuques du phosphore du plasma et des fèces chez les trois béliers, après injection de ${ }^{\mathbf{3 2}} \mathrm{P}$

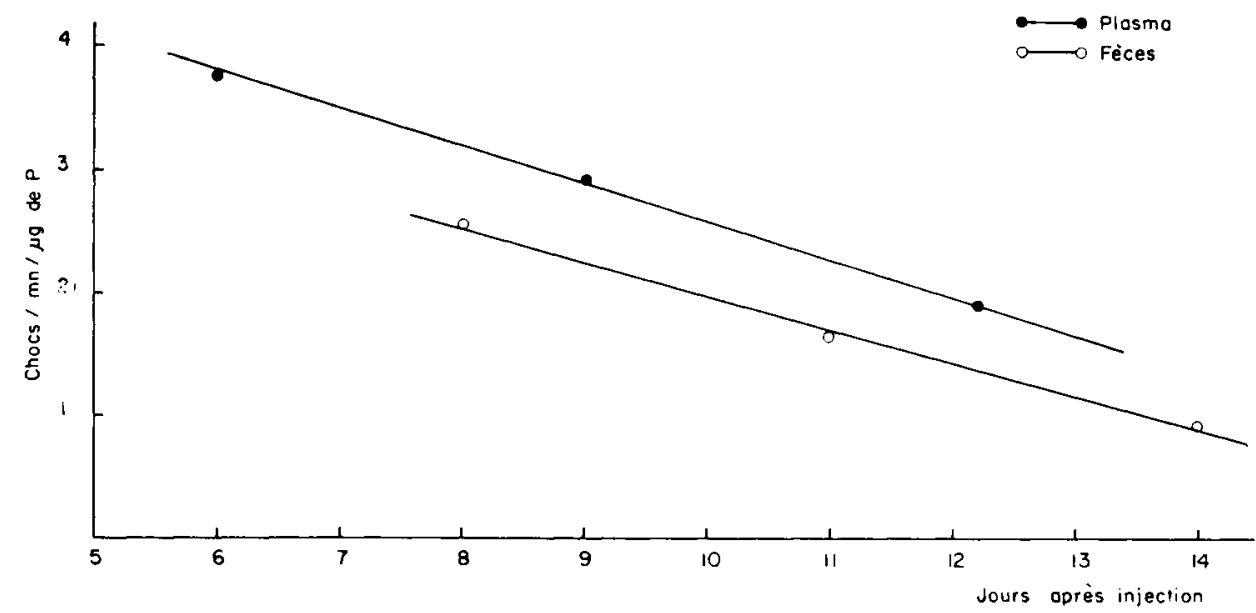

Fig. 2. - Évolution des radioactivités spécifiques moyennes du phospore du plasma et des fèces du $6^{\mathrm{e}}$ all $\mathrm{I} 4^{\mathrm{e}}$ jour après l'injection de ${ }^{\mathbf{3 2}} \mathrm{P}$

Comme l'avaient montré LOFGREEN et KL,EIBER (I954) les décroissances sont pratiquement linéaires durant la période allant $\mathrm{du} 7^{\mathrm{e}}$ au $\mathrm{I}_{4}^{\mathrm{e}}$ jour après l'injection (fig. 2). Il est donc possible de se limiter à cette période pour effectuer les calculs. 


\section{$2^{\circ}$ Évaluation de la proportion de phosphore endogène dans le phosphore fécal total}

Le rapport moyen des activités spécifiques du phosphore des fèces et du plasma, donnant la proportion de $\mathrm{P}$ endogène dans le $\mathrm{P}$ fécal total, a été calculé, d'une part durant la période de 6 jours entre le $\mathrm{I}^{\mathrm{er}}$ et le $3^{\mathrm{e}}$ prélèvement (méthode préconisée par L,OFGREEN et KLEIBER, I954), d'autre part, en faisant la moyenne des résultats obtenus pour les quatre prélèvements. Dans le premier cas les valeurs obtenues pour les 3 animaux $\mathrm{A}, \mathrm{B}$, et $\mathrm{C}$ sont respectivement de $0,56,0,59$ et 0,62 . Dans le second cas les rapports sont de $0,57,0,57$ et 0,62 . Le fait d'utiliser une période de 6 jours et un nombre limité de prélèvements semble donc très valable.

Ainsi, en moyenne, $59 \mathrm{p}$. Ioo du phosphore excrété par les fèces chez le mouton adulte à l'état d'entretien est endogène.

\section{TABLEAU 2}

Radioactivités spécifiques du phosphore du plasma et des fèces (exprimées en chocs/mn/mg de P).

\begin{tabular}{|c|c|c|c|c|}
\hline Animaux & Périodes & Plasma & Fèces & $\frac{\text { Fèces }}{\text { Plasma }}$ \\
\hline \multirow{4}{*}{ A } & 1 & 4330 & 2800 & 0,65 \\
\hline & $\underline{z}$ & 3380 & 1770 & 0,52 \\
\hline & 3 & 2120 & 1030 & 0,49 \\
\hline & 4 & 900 & 5,0 & 0,60 \\
\hline \multirow{4}{*}{ B } & 1 & 2890 & 1920 & 0,66 \\
\hline & 2 & 2130 & 1190 & 0,56 \\
\hline & $\because$ & 1320 & 630 & 0,48 \\
\hline & 4 & 520 & 340 & 0,65 \\
\hline \multirow{4}{*}{$\mathrm{C}$} & 1 & 4080 & $3 \longdiv { 2 0 0 }$ & 0,73 \\
\hline & 2 & 3210 & 1910 & 0,59 \\
\hline & 3 & 2160 & 1120 & 0,52 \\
\hline & 4 & 940 & 600 & 0,64 \\
\hline
\end{tabular}

\section{$3^{\circ}$ Bilan et utilisation digestive réelle du phosphore du foin de luzerne}

Durant la période étudiée les animaux sont en bilan faiblement négatif (tabl. 3) et le coefficient d'utilisation digestive apparent moyen est inférieur à zéro. Comme chez tous les ruminants adultes, l'excrétion urinaire de $\mathrm{P}$ est très faible.

TABLEAU 3

Bilans du phosphore

\begin{tabular}{|c|c|c|c|c|}
\hline Animaux & A & B & $\mathrm{C}$ & Moyenne \\
\hline Ingéré, g par jour............... & 3,36 & 3,40 & 3,05 & $3,2^{\prime} \dot{t}$ \\
\hline Fécal, g par jour $\ldots \ldots \ldots \ldots \ldots \ldots$ & 3,41 & 3,92 & 3,32 & 3,55 \\
\hline 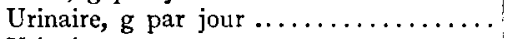 & 0,0 ' & 0,04 & $0,0_{4}^{\prime}$ & $0,0 \dot{t}$ \\
\hline$\frac{\text { Urinaire }}{\text { Fécal }} \times 100 \ldots \ldots \ldots \ldots \ldots \ldots \ldots$ & 1,3 & 1,3 & 1,4 & 1,3 \\
\hline Absorbé apparent, g par jour ........ & $\longrightarrow 0,15$ & $-0,52$ & $-0,27$ & $-0,31$ \\
\hline Retenu apparent, g par jour ......... & $-0,19$ & $-0,56$ & $-0,31$ & $-0,35$ \\
\hline C. U. D. apparent, p. $100 \ldots \ldots \ldots \ldots$ & $<0$ & $<0$ & $<0$ & $<0$ \\
\hline Endogène fécal, g par jour........... & $1, \overline{93}$ & 2,27 & 2,05 & 2,08 \\
\hline Exogène fécal, g par jour............. & 1,48 & 1,65 & 1,27 & 1,47 \\
\hline Absorbé réel, $\mathrm{g}$ par jour ............. & 1,77 & 1,75 & 1,77 & 1,76 \\
\hline C. U. D. réel, p. $100 \ldots \ldots \ldots \ldots \ldots \ldots$ & 54,7 & 52,1 & 57,9 & $5^{\prime}, 9$ \\
\hline
\end{tabular}


'Toutefois, l'excrétion endogène fécale de $\mathrm{P}$ étant élevée (en moyenne 2 , I g par jour) les quantités réelles absorbées sont en moyenne de $I, 76 \mathrm{~g}$ par jour et très voisines chez les 3 animaux. Ainsi le coefficient d'utilisation digestive réel moyen est de 54,9 p. IOO.

Puisque l'excrétion urinaire de $\mathrm{P}$ est négligeable ( $\mathrm{c}$ p. roo de l'excrétion totale) l'excrétion fécale endogène de $P$ fournit la totalité des pertes métaboliques. Le besoin d'entretien peut donc être estimé à $2, \mathrm{I} \mathrm{g}$ de $\mathrm{P}$ par jour. Compte tenu du CUD réel de $55 \mathrm{p}$. IOO, ceci conduirait à recommander l'apport journalier de $3,8 \mathrm{~g}$ de $\mathrm{P}$.

\section{DISCUSSION}

La méthode de dilution isotopique employée pour évaluer l'excrétion fécale endogène de $\mathrm{P}$ semble particulièrement fidèle et les évolutions des activités spécifiques de $\mathrm{P}$ du plasma et des fèces sont remarquablement similaires. Comme l'avaient préconisé LOFGREEN et KLEIBER (I954) il se semble pas nécessaire d'effectuer des prélèvements très nombreux, compte tenu de l'existence d'une période de plusieurs jours durant laquelle la diminution des radioactivités spécifiques de $\mathrm{P}$ du plasma et des fèces est linéaire.

Certains auteurs rapportent l'activité spécifique de $\mathrm{P}$ des fèces à l'activité spécifique de P de l'urine, ce qui constitue une simplification considérable (WRIGHT I955, GÜTTE et al., Ig6I). Toutefois, cette technique serait surtout utilisable chez les monogastriques, oì 1'excrétion urinaire de ${ }^{32} \mathrm{P}$ est élevée. Chez les ruminants il ne semble pas exister de relation continue entre les excrétions urinaires et fécales de ${ }^{32} \mathrm{P}$.

La proportion de $\mathrm{P}$ fécal endogène dans le $\mathrm{P}$ fécal total de $59 \mathrm{p}$. Ioo est intermédiaire entre les deux valeurs de 43 et 70 p. Ioo trouvées par KLEIBER et al. (I95I) sur deux vaches laitières consommant des quantités très différentes de matière sèche sous forme de foin de luzerne et d'aliment concentré.

Les bilans négatifs observés durant la période expérimentale peuvent résulter de l'insuffisance des quantités de $P$ ingérées : les moutons ne recevaient que du foin, et en quantité limitée ( $\mathrm{I} 500 \mathrm{~g}$ de matière sèche par jour en moyenne). Cependant, la digestibilité réelle de $\mathrm{P}$ du foin de luzerne est élevée $(55 \mathrm{p}$. Ioo) et proche de la valeur de $64 \mathrm{p}$. Ioo trouvée par BR̈̈GGEMAN et al. (I959) sur des moutons adultes recevant exclusivement du foin et qui se trouvaient également en bilan négatif. KLEIBER et al. (I95) sur deux vaches recevant un foin de luzerne et un aliment concentré trouvent que 50 et $64 \mathrm{p}$. Ioo du $\mathrm{P}$ de la ration totale est absorbé, tandis que WRIGH'T (I955), travaillant sur brebis, indique des digestibilités réelles de $5^{6}$ et 82 p. roo pour le $P$ d'une ration comprenant un foin mixte et un aliment concentré.

Le besoin d'entretien, estimé à $2, \mathrm{I} g$ de $\mathrm{P}$ par jour, est couvert par un apport journalier de $3,8 \mathrm{~g}$ de $P$, soit $42 \mathrm{mg}$ de $\mathrm{P}$ par $\mathrm{kg}$ de poids vif. L'apport habituellement recommandé chez les bovins adultes est de $30 \mathrm{mg}$ par jour et par kg de poids vif.

Compte tenu de l'ingestion moyenne de $\mathrm{I} .500 \mathrm{~g}$ de matière sèche par jour, la teneur en $P$ recommandée dans la ration totale serait donc de 2,5 g de $P$ par $\mathrm{kg}$ de matière sèche. Cependant, des rations différentes conduiraient à des consommations normales plus élevées ( $2 \mathrm{~kg}$ de matière sèche par jour) et il suffirait alors de $2 \mathrm{~g}$ de $\mathrm{P}$ par $\mathrm{kg}$ de matière sèche pour couvrir les besoins d'entretien. 


\section{SUMMARY}

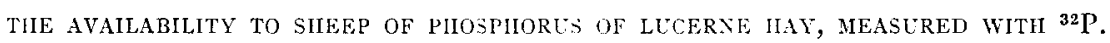

The faecal metabolic excretion of phosphorus has been estimated in three adult rams by a simplified method of isotopic dilution according to LOFGREEN and KLEIBER. The balance of phosphorus were effected from the 6 th day after a single injection of ${ }^{32} \mathrm{P}$ and the specific activities of phosphorus of the plasma and the faeces have been compared.

The specific activities of phosphorus of the plasma and the faeces followed a linear decrease from the 6th to the $\mathrm{I} 4 \mathrm{th}$ day after the injection and the parallelism between the three animals was remarkable. In order to verify the validity of this simplified method the experiment was extended to the 2Ist day (fig. I and 2).

On an average, $60 \mathrm{p}$. 100 of the phosphorus excreted by the faeces was endogenous. Thus, the true digestibility of the phosphorus of the lucerne hay distributed was $55 \mathrm{p}$. . 00 , while its apparent digestibility was negative (tab. 3 ).

The faecal metabolic excretion of phosphorus was $2.6 \mathrm{~g}$ per day; this constitutes approximately the maintenance requirement since the urinary loss of phosphorus is negligible (about I p. Ioo of the total excretion).

'To cover the maintenance requirement, $3.8 \mathrm{~g}$ of phosphorus should be given per day. This corresponds to $42 \mathrm{mg}$ of phosphorus per $\mathrm{kg}$ live weight of the animal and about $2.5 \mathrm{~g}$ of phosphorus per kg dry matter fed.

\section{RÉFÉRENCES BIBLIOGRAPHIQUES}

Brügremaix J., Bronsch K., Lörcher K., SEYss II., I959. Die Bestimnung der I'hosphorressorption aus Mineralfuttem. Zeitschr. Tierphys. Tieremällr. Fitlermilt., 14, 22+-24I.

Gütte J. O., Laytzsci H.J., MolNár S., Lexkeit W., ig6r. Veränderungen der intestinalen PhosphorAbsorption und - Exkretion im Verlauf der Gravidiät und laktation des Schweines bei konstanter Ernährung. Zeitschr. Tierphys. Tievernähr. Futtermitt., 16, $75-90$.

IIEvesy G., HAIN L., ReBbe O., I939. Excretion of phosphorus. Det. Kgl. Danske Vilenskabernes Selskab. Biologiske Meddelelser, Kobenhavn, 14, $\mathrm{n}^{\circ} 3$.

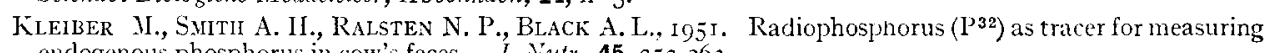
endogenous phosphorus in cow's feces. J. Nutr., 45, $253-263$.

LoFGREEN G. P., I 960 . The availability of the phosphorus in dicalcium phosphate, bone meal, soft phosphate and calcium phytate for mature wethers. J. Nutr., 70, $58-62$.

LOFGREEN G. P., KLEIBER M., I954. Further studies on the availability of phosphorus in alfalfa hay. $J$. Anim. Sci., 13, $258-264$.

WRIGUT E., 1955. The uptake of phosphorus by the sheep. I. The excretion of injected radiophosphorus. 2. The availability of the phosphorus of a diet of hay and concentrates. N.Z.J. Sci. Technol. (A), 37,332$34^{8}$. 